\title{
RIGHT-CANCELLABLE PROTOMODULAR ALGEBRAS
}

\author{
DALI ZANGURASHVILI
}

\begin{abstract}
A new protomodular analog of the classical criterion for the existence of a group term in the algebraic theory of a variety of universal algebras is given. To this end, the notion of a right-cancellable protomodular algebra is introduced. It is proved that the algebraic theory of a variety of universal algebras contains a group term if and only if it contains protomodular terms with respect to which all algebras from the variety are right-cancellable. This, in particular, gives a partial answer to the extended version of an open problem from loop theory whether any Hausdorff topological (semi-)loop is completely regular. Moreover, the rightcancellable algebras from the simplest protomodular varieties are characterized as sets with principal group actions as well as groups with simple additional structures.
\end{abstract}

Key words and phrases: protomodular variety; right-cancellable protomodular algebra; group term.

2020 Mathematics Subject Classification: 18C05, 08B05, 22A30, $20 \mathrm{~N} 05$.

\section{INTRODUCTION}

It is well-known that the algebraic theory of a variety of universal algebras contains a group term (i.e. a binary term that satisfies the group identities for some unary and 0-ary terms) if and only if it contains a 0-ary term and an associative Mal'cev term, i.e. a Mal'cev term that satisfies the identity:

$$
p(x, t, p(s, y, z))=p(p(x, t, s), y, z) .
$$

This is explained e.g. in [7] and in [13].

Recall that one of important classes of Mal'cev varieties is that of protomodular varieties. The notion of a protomodular variety, and, more generally, of a protomodular category was introduced by Bourn in [5] as an abstract setting where certain properties of groups remain valid, and, in particular, there is an intrinsic notion of a Bourn normal subobject. The class of protomodular categories contains (but does not coincides with) the class of semi-abelian categories where one can 
develop isomorphism and decomposition theorems, radical and commutator theory, homology of non-abelian structures [11].

There is a syntactical characterization of protomodular varieties due to Bourn and Janelidze [6]. It requires the existence of a term $\theta$ of an arbitrarily high arity $(n+1)$, binary terms $\alpha_{i}$ and 0 -ary terms $e_{i}(i=$ $1,2, \ldots, n$ ) that satisfy certain identities (which, in fact, first appeared in Ursini's paper [14, but for purely universal-algebraic reasons and only in the particular case were all $e_{i}$ 's are equal to one another). For convenience, we call these terms protomodular.

In the previous paper [16] we give a protomodular analog of the above-mentioned criterion for the existence of a group term in a variety of universal algebras. It requires a certain kind of higher associativity condition on a protomodular term $\theta$ and the fulfillment of the identities $e_{1}=e_{2}=\ldots=e_{n}$. In fact, in [16] we also give the second protomodular analog of that criterion, but it involves a rather cumbersome identity.

In the present paper we give yet another criterion for the existence of a group term in a variety of universal algebras. It is also formulated in protomodular terms, but, as different from our first criterion, involves no requirement related to 0 -ary terms, and, moreover, the identitites involved in it are simpler than the identity from our second criterion.

For the proposed criterion we introduce the notion of a right - cancellable protomodular algebra (with respect to protomodular terms $\theta$ and $\alpha_{i}$ ). We define it as a protomodular algebra that satisfies the additional identities

$$
\begin{aligned}
& \alpha_{i}\left(\theta\left(a_{1}, a_{2}, \ldots, a_{n}, b\right), \theta\left(a_{1}^{\prime}, a_{2}^{\prime}, \ldots, a_{n}^{\prime}, b\right)\right)= \\
= & \alpha_{i}\left(\theta\left(a_{1}, a_{2}, \ldots, a_{n}, b^{\prime}\right), \theta\left(a_{1}^{\prime}, a_{2}^{\prime}, \ldots, a_{n}^{\prime}, b^{\prime}\right)\right),
\end{aligned}
$$

for all $i=1,2, \ldots, n$ (and follow this definition throughout the paper, in spite of the fact that for $n=1$ it is not equivalent to the traditional one of right-cancellability $(a b=c b \Rightarrow a=c))$.

It is proved that for any element $u$ of a right-cancellable algebra $A$ the binary operation defined by

$$
a \cdot{ }_{u} b=\theta\left(\alpha_{1}(a, u), \alpha_{2}(a, u), \ldots, \alpha_{n}(a, u), b\right)
$$

is a group operation with unit $u$ and the inverses given by

$$
a^{-{ }_{u}^{1}}=\theta\left(\alpha_{1}(u, a), \alpha_{2}(u, a), \ldots, \alpha_{n}(u, a), u\right) .
$$

This fact yields the new protomodular analog of the above-mentioned group term existence criterion that reads as follows: 
The algebraic theory of a variety of universal algebras contains a group term if and only if it contains protomodular terms with respect to which all algebras from this variety are right-cancellable.

It should be noted that the Mal'cev operation corresponding to the group operation (1.3) coincides with that given by Bourn and Janelidze in [6], and by Ursini in [15. Hence the fact that (1.3) is a group operation implies in particular that this Mal'cev operation is associative on right-cancellable protomodular algebras.

Note also that the fact that (1.3) is a group operation relates to an open problem from loop theory whether the classical result of topological group theory by which any Hausdorff topological group is completely regular holds true for loops too ([8], Problem IX.1.17). Although our result does not provide a solution of this problem as such (because, as is shown, any loop which is right-cancellable in the sense of this paper is a group), nevertheless it gives a partial answer to the extended version of the problem, namely whether any Hausdorff topological left semi-loop is completely regular. It is shown that the class of left semi-loops which are not groups, but for which the answer to the latter problem is positive is quite wide: such left semi-loops can be obtained in a simple way from arbitrary groups $G$ equipped with any non-trivial bijections $\sigma: G \rightarrow G$ (that are not necessarily automorphisms). This is accomplished by characterizing the right-cancellable algebras from the simplest protomodular varieties (i.e. the varieties where the signature contains only protomodular operations, while the defining identities are precisely identities (2.1) and (2.2)) as groups with simple additional structures. Such algebras are characterized also as sets with principal group actions.

\section{Preliminaries}

For the definition of a protomodular category we refer the reader to the paper [5] by Bourn.

Let $\mathbb{V}$ be a variety of universal algebras of type $\mathcal{F}$.

Theorem 2.1. (Bourn-Janelidze [6]) A variety $\mathbb{V}$ is protomodular if and only if its algebraic theory contains, for some natural $n$, 0-ary terms $e_{1}, e_{2}, \ldots, e_{n}$, binary terms $\alpha_{1}, \alpha_{2}, \ldots, \alpha_{n}$, and an $(n+1)$-ary term $\theta$ such that the following identities are satisfied:

$$
\begin{gathered}
\alpha_{i}(a, a)=e_{i} ; \\
\theta\left(\alpha_{1}(a, b), \alpha_{2}(a, b), \ldots, \alpha_{n}(a, b), b\right)=a .
\end{gathered}
$$


For simplicity, algebras from a protomodular variety are called protomodular algebras. The terms $\theta, \alpha_{i}$ and $e_{i}$ satisfying (2.1) and (2.2) are called protomodular.

The motivating example of a protomodular variety is given by the variety of groups. More generally, any variety whose algebraic theory contains a group term is protomodular (which in particular implies that the variety of Boolean algebras is protomodular). In that case we have:

$$
\begin{aligned}
\theta(a, b) & =a b, \\
\alpha_{1}(a, b) & =a / b .
\end{aligned}
$$

and $e$ is unit of the group. Note that the variety of Boolean algebras also has another system of protomodular terms [4] which are given by:

$$
\begin{gathered}
\theta(a, b, c)=(a \vee c) \wedge b, \\
\alpha_{1}(a, b)=a \wedge \neg b, \\
\alpha_{2}(a, b)=a \vee \neg b,
\end{gathered}
$$

with $e_{1}=0$ and $e_{2}=1$.

Other examples of protomodular varieties are provided by the varieties of left/right semi-loops, loops, locally Boolean distributive lattices [3], Heyting algebras, Heyting semi-lattices [12]. Observe that the terms (2.3) and (2.4) serve as protomodular terms in the case of left semi-loops and loops, too.

Let $\mathbb{V}$ be a protomodular variety of universal algebras. Identities (2.1) and (2.2) immediately imply [6], [15, [4] the following:

(a) if $\alpha_{i}(a, c)=\alpha_{i}(b, c)$, for all $i(1 \leq i \leq n)$, then $a=b$;

(b) if $\alpha_{i}(a, b)=e_{i}$, for all $i(1 \leq i \leq n)$, then $a=b$;

(c) the identity

$$
\theta\left(e_{1}, e_{2}, \ldots, e_{n}, a\right)=a
$$

(d) the term

$$
p(a, b, c)=\theta\left(\alpha_{1}(a, b), \alpha_{2}(a, b), \ldots, \alpha_{n}(a, b), c\right) .
$$

is a Mal'cev term.

If, in addition, $e_{1}=e_{2}=\ldots=e_{n}$, then $\mathbb{V}$ is ideal determined [15]. Recall that this means that any ideal of any $\mathbb{V}$-algebra is the kernel of a unique congruence (Gumm-Ursini [10]). Moreover, for any congruence $R$ of an $\mathbb{V}$-algebra $A$, the equivalence class containing an element $a \in A$ is equal to the set $\theta(I, I, \ldots, I, a)$ of all elements of the form $\theta\left(b_{1}, b_{2}, \ldots, b_{n}, a\right)$, where $b_{1}, b_{2}, \ldots, b_{n} \in I$, while $I$ is the kernel of $R$ [2]. 
Let $A$ be a set. For any element $u \in A$, there is a bijection between the set of associative Mal'cev operations on $A$ and the set of group operations on $A$ with unit $u$. The bijection is given as follows: if $p$ is an associative Mal'cev operation, then the group operation is given by $a b=p(a, u, b)$ with $a^{-1}=p(u, a, u)$. If $\cdot$ is a group operation with unit $u$, then the corresponding Mal'cev operation is given by $p(a, b, c)=$ $a b^{-1} c$ [13] (see e.g. [7] and[13]).

Let as above $A$ be a set and let $\theta$ be an $(n+1)$-ary operation on $A$. The operation $\theta$ is called 1-associative [16] if by shifting the internal symbol $\theta$ together with the attached parentheses in the expression

$$
\theta\left(a_{1}, a_{2}, \ldots, a_{n}, \theta\left(b_{1}, b_{2}, \ldots, b_{n}, c\right)\right)
$$

to any position, the value of the expression does not change, for any $a_{1}, a_{2}, \ldots, a_{n}, b_{1}, b_{2}, \ldots, b_{n}, c \in A$.

The operation $\theta$ is called 2-associative (or consociative) [16] if the equality

$$
\begin{gathered}
\theta\left(a_{1}, a_{2}, \ldots, a_{n}, \theta\left(b_{1}, b_{2}, \ldots, b_{n}, c\right)\right)= \\
\theta\left(\theta\left(a_{1}, a_{2}, \ldots, a_{n}, b_{1}\right), \theta\left(a_{1}, a_{2}, \ldots, a_{n}, b_{2}\right), \ldots, \theta\left(a_{1}, a_{2}, \ldots, a_{n}, b_{n}\right), c\right)
\end{gathered}
$$

holds for any $a_{1}, a_{2}, \ldots, a_{n}, b_{1}, b_{2}, \ldots, b_{n}, c \in A$. This definition is motivated by the fact that, for a binary operation $\times$ on $A$, the associativity can be formulated as the condition that the mapping $(-\times a)$ from $A$ to the algebra $\operatorname{Map}(A, A)$ of mappings $A \rightarrow A$ (with the composition operation) preserves the operation $\times$, for any $a \in A$. Generalizing this condition to the case of an operation $\theta$ of high arity, we obtain precisely identity (2.10).

For ternary operations, the 1-associativity implies associativity (1.1) (below we will refer to the latter condition as "associativity"). No other implications hold between the above three associativity(-like) conditions - associativity, 1-associativity, and 2-associativity [16].

A protomodular algebra is called 1-associative (resp. 2-associative or consociative) if its protomodular operation $\theta$ is such.

A protomodular term $\theta$ is called 2-associative or consociative if it satisfies identity (2.10).

Let $A$ be a protomodular algebra and $b \in A$. We denote by $\theta_{b}$ the mapping $A^{n} \rightarrow A$ sending an $n$-tuple $\left(a_{1}, a_{2}, \ldots, a_{n}\right)$ to $\theta\left(a_{1}, a_{2}, \ldots, a_{n}, b\right)$.

A protomodular algebra $A$ is called strict [16] if $\theta_{b}$ is a bijection, for any $b \in A$, or equivalently, if the following identity holds in $A$ :

$$
\alpha_{i}\left(\theta\left(a_{1}, a_{2}, \ldots, a_{n}, b\right), b\right)=a_{i}
$$

for any $i(i=1,2, \ldots, n)$. 
Throughout the paper $\mathbb{V}_{n}$ denotes the simplest protomodular variety, i.e., the variety with the signature $\mathcal{F}_{n}$ containing only one $(n+1)$-ary operation symbol $\theta$, the binary operation symbols $\alpha_{1}, \alpha_{2}, \ldots, \alpha_{n}$, and the 0 -ary operation symbols $e_{1}, e_{2}, \ldots, e_{n}$, where the defining identities are (2.1) and (2.2).

Strict $\mathbb{V}_{1}$-algebras are precisely left semi-loops [16].

\section{Right-CAnCEllable Protomodular Algebras}

Definition 3.1. Let $\mathbb{V}$ be a protomodular variety, and $A$ be its algebra. A is called right-cancellable with respect to protomodular terms $\theta$ and $\alpha_{i}$ (or simply right-cancellable if no confusion might arise) if the identity

$$
\begin{aligned}
& \alpha_{i}\left(\theta\left(a_{1}, a_{2}, \ldots, a_{n}, b\right), \theta\left(a_{1}^{\prime}, a_{2}^{\prime}, \ldots, a_{n}^{\prime}, b\right)\right)= \\
& =\alpha_{i}\left(\theta\left(a_{1}, a_{2}, \ldots, a_{n}, b^{\prime}\right), \theta\left(a_{1}^{\prime}, a_{2}^{\prime}, \ldots, a_{n}^{\prime}, b^{\prime}\right)\right),
\end{aligned}
$$

holds in $A$ for any $i(1 \leq i \leq n)$.

Example 3.2. Groups considered as protomodular algebras obviously are right-cancellable. Moreover, there are left semi-loops which are not groups, but are right-cancellable. Take for instance the set $A=\{0,1,2\}$ with the following binary operations $\theta$ and $\alpha$ equal to each other:

\begin{tabular}{|c|ccc|}
\hline$\theta=\alpha$ & 0 & 1 & 2 \\
\hline 0 & 0 & 1 & 2 \\
1 & 2 & 0 & 1 \\
2 & 1 & 2 & 0 \\
\hline
\end{tabular}

One can easily verify that $A$ is a right-cancellable left semi-loop.

It should be however noted that not all left semi-loops are rightcancellable. For instance, the (transpose of the right) semi-loop given in the example of [1] is not right-cancellable.

Example 3.3. Let $A=\{0,1\}$. Let us introduce the structure of $\mathbb{V}_{2^{-}}$ algebra on $A$ as follows. Let $\theta(i, j, k)=k$ if $i \neq j$ and $\theta(i, j, k)=1-k$ if $i=j$. Moreover, let $\alpha_{1}(i, j)$ be 0 , for any $i, j$; let $\alpha_{2}(i, j)$ be 0 , if $i \neq j$, and be 1 , if $i=j$. Besides, let $e_{1}=0$ and $e_{2}=1$.

The condition (3.1) is obviously satisfied for $i=1$. It also is satisfied for $i=2$ since the value of the left-hand side of (3.1) depends only on whether $a_{1}=a_{2}$ and $a_{1}^{\prime}=a_{2}^{\prime}$. This implies that $A$ is a right-cancellable $\mathbb{V}_{2}$-algebra. 
Example 3.4. Let $A=\{0,1,2,3\}$, and $n=3$. We define operations on the set $A$ by means of the tables below, where $t_{0}$ is a triple $\left(a_{1}, a_{2}, a_{3}\right)$ from $A^{3}$ with $a_{1}, a_{2}, a_{3} \leq 1, t_{1}$ is a triple with $a_{1} \leq 1$ and either $a_{2}>1$ or $a_{3}>1, t_{2}$ is a triple with $a_{1}>1$ and either $a_{2} \leq 1$ or $a_{2} \leq 1$, while $t_{3}$ is a triple with $a_{1}, a_{2}, a_{3}>1$.

\begin{tabular}{|c|llll|}
\hline$\theta$ & 0 & 1 & 2 & 3 \\
\hline$t_{0}$ & 0 & 1 & 2 & 3 \\
$t_{1}$ & 1 & 0 & 3 & 2 \\
$t_{2}$ & 2 & 3 & 0 & 1 \\
$t_{3}$ & 3 & 2 & 1 & 0 \\
\hline
\end{tabular}

\begin{tabular}{|c|cccc|}
\hline$\alpha_{1}$ & 0 & 1 & 2 & 3 \\
\hline 0 & 0 & 1 & 3 & 2 \\
1 & 1 & 0 & 2 & 3 \\
2 & 3 & 2 & 0 & 1 \\
3 & 2 & 3 & 1 & 0 \\
\hline
\end{tabular}

\begin{tabular}{|c|cccc|}
\hline$\alpha_{2}$ & 0 & 1 & 2 & 3 \\
\hline 0 & 0 & 2 & 0 & 3 \\
1 & 2 & 0 & 3 & 0 \\
2 & 0 & 3 & 0 & 2 \\
3 & 3 & 0 & 2 & 0 \\
\hline
\end{tabular}

\begin{tabular}{|c|cccc|}
\hline$\alpha_{3}$ & 0 & 1 & 2 & 3 \\
\hline 0 & 1 & 1 & 2 & 2 \\
1 & 1 & 1 & 2 & 2 \\
2 & 2 & 2 & 1 & 1 \\
3 & 2 & 2 & 1 & 1 \\
\hline
\end{tabular}

Let $e_{1}=e_{2}=0$ and $e_{3}=1$. It can be verified that the set $A$ equipped with these operations is a right-cancellable $\mathbb{V}_{3}$-algebra.

At the end of Section 5 we will show how to construct right-cancellable $\mathbb{V}_{n}$-algebras of an arbitrary cardinality, for any $n$.

Below, when dealing with the case $n=1$, we will use the traditional abbreviation $a b$ for $\theta(a, b)$, and $a / b$ for $\alpha(a, b)$.

Lemma 3.5. Let $\mathbb{V}$ be a protomodular variety with $n=1$, and let $A$ be an algebra from this variety with unit e. The following conditions are equivalent:

(i) A is right-cancellable and, for any $a \in A$, we have:

$$
a e=a ;
$$

(ii) A is right-cancellable and, for any $a \in A$, we have:

$$
a / e=a
$$

(iii) $A$ is a group.

Proof. (i) $\Rightarrow($ ii): From (2.2) we have $(a / e) e=a$; on the other hand, (3.5) implies $(a / e) e=a / e$.

(i) $\Rightarrow$ (iii): From (3.5) we obtain the identity

$$
(a b) /\left(a^{\prime} b\right)=a / a^{\prime} .
$$


We already know that identity (3.5) implies (3.6). Hence from (3.7) we obtain

$$
(a b) c /(b c)=(a b) / b=a b / e b=a / e=a,
$$

for any $a, b, c \in A$. Multiplying both parts of this equality by $(b c)$ from the right, we obtain the associativity condition. Then taking into account the fact that any set equipped with an associative binary operation that has a left identity and left inverses, is a group (see, e.g. [9]), we obtain that $A$ is a group.

The implications (ii) $\Rightarrow$ (i) and (iii) $\Rightarrow$ (i) are obvious.

In particular, we obtain

Proposition 3.6. Any loop which is right-cancellable in the sense of Definition 3.1 is a group.

Proposition 5.7 that will be given at the end of the paper implies

Proposition 3.7. Let $\mathbb{V}$ be a protomodular variety with $n=1$. Any finite $\mathbb{V}$-algebra which is right-cancellable in the sense of Definition 3.1 is a left semi-loop.

Remark 3.8. An algebra can be right-cancellable with respect to one system of protomodular terms, but might be not such with respect to some other system of protomodular terms. The example is provided by non-trivial Boolean algebras. They obviously are right-cancellable with respect to the protomodular terms determined by a group term of the variety of Boolean algebras. However, these algebras are not right-cancellable with respect to the protomodular terms (2.5)-(2.7), as follows from the equality $\alpha_{2}(\theta(0,0, b), b)=\neg b$, and identity (2.8).

Remark 3.9. It can be shown that non-trivial locally Boolean distributive algebras are not right-cancellable with respect to the protomodular terms given in [4. Similarly, non-trivial Heyting algebras and non-trivial Heyting semi-lattices are not right-cancellable with respect to the protomodular terms given in [12] .

Identities (3.1) can be simplified in some cases. More precisely, we have

Lemma 3.10. Let $A$ be a protomodular algebra. For the conditions below we have the implications $(i) \Rightarrow($ ii) and (i) $\Rightarrow$ (iii). If $A$ is either 1-associative or 2-associative, then the conditions (i) and (ii) are equivalent:

(i) A is right-cancellable; 
(ii) the identity

$$
\begin{aligned}
& \alpha_{i}\left(b, \theta\left(a_{1}, a_{2}, \ldots, a_{n}, b\right)\right)= \\
= & \alpha_{i}\left(b^{\prime}, \theta\left(a_{1}, a_{2}, \ldots, a_{n}, b^{\prime}\right)\right),
\end{aligned}
$$

holds in $A$ for any $i(1 \leq i \leq n)$;

(iii) the identity

$$
\begin{aligned}
& \alpha_{i}\left(\theta\left(a_{1}, a_{2}, \ldots, a_{n}, b\right), b\right)= \\
= & \alpha_{i}\left(\theta\left(a_{1}, a_{2}, \ldots, a_{n}, b^{\prime}\right), b^{\prime}\right),
\end{aligned}
$$

holds in $A$ for any $i(1 \leq i \leq n)$.

Proof. The implications (i) $\Rightarrow$ (ii) and (i) $\Rightarrow$ (iii) immediately follow from identity (2.8).

(ii) $\Rightarrow($ i): From $(2.2)$ we have

$$
\begin{gathered}
\alpha_{i}\left(\theta\left(a_{1}, a_{2}, \ldots, a_{n}, b\right), \theta\left(a_{1}^{\prime}, a_{2}^{\prime}, \ldots, a_{n}^{\prime}, b\right)\right)= \\
\alpha_{i}\left(\theta\left(a_{1}, a_{2}, \ldots, a_{n}, b\right), \theta\left(a_{1}^{\prime}, a_{2}^{\prime}, \ldots, a_{n}^{\prime}, \theta\left(c_{1}, c_{2}, \ldots, c_{n}, \theta\left(a_{1}, a_{2}, \ldots, a_{n}, b\right)\right)\right)\right),
\end{gathered}
$$

where

$$
c_{i}=\alpha_{i}\left(b, \theta\left(a_{1}, a_{2}, \ldots, a_{n}, b\right)\right) .
$$

By (3.5) the value of $c_{i}$ does not depend on $b$. Applying the 1-associativity (resp. the 2-associativity) and then again (3.5), we obtain (3.1).

Remark 3.11. The implication (ii) $\Rightarrow$ (i) does not hold in general. The counterexample is provided by any Moufang loop which is not a group, as follows from Proposition 3.6.

As to the implication (iii) $\Rightarrow(\mathrm{i})$, it is not valid either, as the same counterexample shows. Moreover, we do not impose the additional condition of 1-associativity or 2-associativity on the algebras satisfying (3.6) for the following reason. If $n=1$, then, as is observed in Example 3.15 of [16], the associativity condition on an algebra of this kind implies that it is a group. If $n \geq 2$, then either of the 1-associativity and 2associativity conditions implies that such an algebra is trivial, as is shown in Remark 3.16 of [16].

\section{Right-CANCELlable PRotomodular ALGEBRAS AS SETS WITH PRINCIPAL GROUP ACTIONS}

Let $A$ be an algebra from a protomodular variety, and $b \in A$. Recall that the symbol $\theta_{b}$ denotes the mapping $A^{n} \rightarrow A$ sending an $n$-tuple $\left(a_{1}, a_{2}, \ldots, a_{n}\right)$ to $\theta\left(a_{1}, a_{2}, \ldots, a_{n}, b\right)$. 
Lemma 4.1. Let $A$ be a right-cancellable protomodular algebra. For any $b, b^{\prime} \in A$, the mappings $\theta_{b}$ and $\theta_{b^{\prime}}$ determine one and the same equivalence relation $\sim$ on $A^{n}$.

Proof. Assume

$$
\theta\left(a_{1}, a_{2}, \ldots, a_{n}, b\right)=\theta\left(c_{1}, c_{2}, \ldots, c_{n}, b\right) .
$$

for some $a_{1}, a_{2}, \ldots, a_{n}, c_{1}, c_{2}, \ldots, c_{n} \in A$. Then, for any $i$, we have

$$
\alpha_{i}\left(\theta\left(a_{1}, a_{2}, \ldots, a_{n}, b\right), \theta\left(c_{1}, c_{2}, \ldots, c_{n}, b\right)\right)=e_{i} .
$$

But since the algebra $A$ is right-cancellable, one can replace both $b$ 's in the left-hand side of (4.2) by $b^{\prime}$, and hence we obtain

$$
\alpha_{i}\left(\theta\left(a_{1}, a_{2}, \ldots, a_{n}, b^{\prime}\right), \theta\left(c_{1}, c_{2}, \ldots, c_{n}, b^{\prime}\right)\right)=e_{i} .
$$

Since (4.3) holds for any $i$, from (b) of Section 2 we conclude that

$$
\theta\left(a_{1}, a_{2}, \ldots, a_{n}, b^{\prime}\right)=\theta\left(c_{1}, c_{2}, \ldots, c_{n}, b^{\prime}\right)
$$

Let $A$ be a protomodular algebra, and $a_{1}, a_{2}, \ldots, a_{n} \in A$. We denote by $\theta^{\left(a_{1}, a_{2}, \ldots, a_{n}\right)}$ the mapping $A \rightarrow A$ sending $b \in A$ to $\theta\left(a_{1}, a_{2}, \ldots, a_{n}, b\right)$, and use the term "translation" for a mapping of this kind.

Lemma 4.1 implies

Lemma 4.2. Let $A$ be a right-cancellable algebra. If the actions of two translations coincide on at least one element of $A$, then these translations are equal.

Lemma 4.3. For the conditions below, the implications $(i) \Rightarrow(i i) \Rightarrow$ (iii) hold. If $A$ is strict, then the conditions (i)-(ii) are equivalent, and the n-tuple $\left(c_{1}, c_{2}, \ldots, c_{n}\right)$ satisfying (4.5) is unique.

(i) $A$ is right-cancellable;

(ii) for any $a_{1}, a_{2}, \ldots, a_{n}, b_{1}, b_{2}, \ldots, b_{n} \in A$ there are $c_{1}, c_{2}, \ldots, c_{n} \in A$ such that

$$
\theta^{\left(c_{1}, c_{2}, \ldots, c_{n}\right)} \theta^{\left(b_{1}, b_{2}, \ldots, b_{n}\right)}=\theta^{\left(a_{1}, a_{2}, \ldots, a_{n}\right)} .
$$

(iii) all translations are bijections, and their inverses are also translations.

Proof. $(i) \Rightarrow(i i)$ : Let $x \in A$ and

$$
c_{i}=\alpha_{i}\left(\theta\left(a_{1}, a_{2}, \ldots, a_{n}, x\right), \theta\left(b_{1}, b_{2}, \ldots, b_{n}, x\right)\right)
$$


for any $i$. Then

$$
\theta\left(c_{1}, c_{2}, \ldots, c_{n}, \theta\left(b_{1}, b_{2}, \ldots, b_{n}, x\right)\right)=\theta\left(a_{1}, a_{2}, \ldots, a_{n}, x\right) .
$$

Since $A$ is right-cancellable, the value of $c_{i}$ does not depend on $x$. This implies (4.5).

$($ ii $) \Rightarrow($ iii $)$ : Taking $a_{1}=e_{1}, a_{2}=e_{2}, \ldots, a_{n}=e_{n}$, and applying $(2.8)$ we obtain

$$
\theta^{\left(c_{1}, c_{2}, \ldots, c_{n}\right)} \theta^{\left(b_{1}, b_{2}, \ldots, b_{n}\right)}=1_{A},
$$

for some $c_{1}, c_{2}, \ldots, c_{n} \in A$. This implies that $\theta^{\left(b_{1}, b_{2}, \ldots, b_{n}\right)}$ is injective. Since $b_{1}, b_{2}, \ldots, b_{n}$ are arbitrary, $\theta^{\left(c_{1}, c_{2}, \ldots, c_{n}\right)}$ is injective too. Then (4.8) implies that $\theta^{\left(c_{1}, c_{2}, \ldots, c_{n}\right)}$ is bijective. Hence $\theta^{\left(b_{1}, b_{2}, \ldots, b_{n}\right)}$ also is bijective and $\theta^{\left(c_{1}, c_{2}, \ldots, c_{n}\right)}$ is its inverse.

$(i i) \Rightarrow(i)$ : From the equality (4.5) it follows that (4.7) is satisfied for any $x \in A$. But, since $A$ is strict, (4.7) implies (4.6).

Remark 4.4. Note that the condition (iii) of Lemma 4.3 does not imply $(i)$, even if $A$ is strict. The counterexample is provided by any loop which is not a group, as follows from Proposition 3.6.

Recall that an action of a group $G$ on a set $X$ is called transitive if, for any $x, y \in X$ there is an element $g \in G$ with $g x=y$. If, moreover, such $g$ 's are unique, or, equivalently, the stabilizer of any element of $X$ is trivial, then we say that the action is sharply transitive or principal. In that case $G$ obviously is bijective to $X$.

Let $A$ be a right-cancellable protomodular algebra, and let $S(A)$ denote the set of all bijections (not necessarily isomorphisms) $A \rightarrow A$. Consider the mapping

$$
\bar{\theta}: A^{n} \rightarrow S(A)
$$

which sends an $n$-tuple $\left(a_{1}, a_{2}, \ldots, a_{n}\right)$ to the bijection $\theta^{\left(a_{1}, a_{2}, \ldots, a_{n}\right)}: A \rightarrow$ A.

Lemma 4.5. Let $A$ be a right-cancellable protomodular algebra. Then the set $\bar{\theta}\left(A^{n}\right)$ is a subgroup of $S(A)$, and its action on $A$ is principal. Moreover, for any $a_{1}, a_{2}, \ldots, a_{n}, b_{1}, b_{2}, \ldots, b_{n} \in A$ and $u \in A$, we have:

(a)

$$
\theta^{\left(a_{1}, a_{2}, \ldots, a_{n}\right)} \theta^{\left(b_{1}, b_{2}, \ldots, b_{n}\right)}=\theta^{\left(c_{1}, c_{2}, \ldots, c_{n}\right)},
$$

where

$$
\begin{gathered}
c_{i}= \\
\alpha_{i}\left(\theta\left(a_{1}, a_{2}, \ldots, a_{n}, u\right), \theta\left(\alpha_{1}\left(u, \theta\left(b_{1}, b_{2}, \ldots, b_{n}, u\right)\right), \alpha_{2}\left(u, \theta\left(b_{1}, b_{2}, \ldots, b_{n}, u\right)\right)\right.\right. \\
\left.\ldots, \alpha_{n}\left(u, \theta\left(b_{1}, b_{2}, \ldots, b_{n}, u\right)\right), u\right)
\end{gathered}
$$


(b)

$$
\left(\theta^{\left(a_{1}, a_{2}, \ldots, a_{n}\right)}\right)^{-1}=\theta^{\left(b_{1}, b_{2}, \ldots, b_{n}\right)}
$$

where

$$
b_{i}=\alpha_{i}\left(u, \theta\left(a_{1}, a_{2}, \ldots, a_{n}, u\right)\right) .
$$

Proof. Identity (2.8) implies that $\bar{\theta}\left(A^{n}\right)$ contains $1_{A}$. Lemma 4.3 implies that $\bar{\theta}\left(A^{n}\right)$ is closed under inverses.

Let us show that $\bar{\theta}\left(A^{n}\right)$ is closed under composition. According to Lemma 4.3, if $f, g \in \bar{\theta}\left(A^{n}\right)$, then $g f^{-1} \in \bar{\theta}\left(A^{n}\right)$. Moreover, $f^{-1} \in$ $\bar{\theta}\left(A^{n}\right)$. Hence $g\left(f^{-1}\right)^{-1}=g f \in \bar{\theta}\left(A^{n}\right)$.

Lemma 4.2 and identity (2.2) imply that the action of $\bar{\theta}\left(A^{n}\right)$ on $A$ is principal.

Equalities (4.11) and (4.12) immediately follow from (4.6) (substitute $a_{i}=e_{i}$ ), while the equalities (4.9) and (4.10) follow from (4.6), (4.11) and (4.12).

Example 4.6. Let $A$ be the protomodular algebra from Example 3.4. One can easily observe that there are precisely four different translations. For instance, the translations $\theta^{(1,2,1)}$ and $\theta^{(3,0,2)}$ are different and non-trivial. Moreover, either of them has order 2. Hence the group $\bar{\theta}\left(A^{n}\right)$ is isomorphic to the Klein Four Group, i.e. the product $\mathbf{Z}_{2} \times \mathbf{Z}_{2}$ of the two cyclic groups of order 2 .

Let $\mathbb{X}_{n}$ denote the category with objects being quadruples

$$
(A, G, \varepsilon, \mu)
$$

where $A$ is a set, $G$ is a group acting on $A$ such that the action is principal, while $\varepsilon$ and $\mu$ are mappings resp. $A^{n} \rightarrow G$ and $G \rightarrow A^{n}$ with

$$
\varepsilon \mu=1_{G} .
$$

Morphisms $(A, G, \varepsilon, \mu) \rightarrow\left(A^{\prime}, G^{\prime}, \varepsilon^{\prime}, \mu^{\prime}\right)$ are pairs $(\varphi, \psi)$, where $\varphi$ is a mapping $A \rightarrow A^{\prime}$, while $\psi$ is a group homomorphism $G \rightarrow G^{\prime}$ such that, for any $g \in G$ and $a \in A$, one has the equality

$$
\varphi(g a)=\psi(g) \varphi(a)
$$

and the diagram

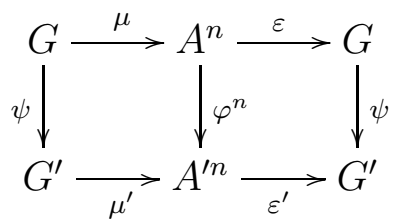

is commutative.

Let $R C-\mathbb{V}_{n}$ be the category of right-cancellable $\mathbb{V}_{n}$-algebras. 
Proposition 4.7. The categories $R C-\mathbb{V}_{n}$ and $\mathbb{X}_{n}$ are equivalent. Under this equivalence the strict $R C-\mathbb{V}_{n}$-algebras correspond precisely to the quadruples $(A, G, \varepsilon, \mu)$ with bijective (and mutually inverse) $\varepsilon$ and $\mu$. If $n=1$, the groups correspond precisely to the quadruples $(A, G, \varepsilon, \mu)$ with

$$
\varepsilon(a) \mu(e)=a
$$

for any $a \in A$.

Proof. Let us construct the functor $F: R C-\mathbb{V}_{n} \rightarrow \mathbb{X}_{n}$ as follows. For a right-cancellable protomodular algebra $A$, take the group $G=\bar{\theta}\left(A^{n}\right)$, and define

$$
\begin{gathered}
\varepsilon\left(a_{1}, a_{2}, \ldots, a_{n}\right)=\theta^{\left(a_{1}, a_{2}, \ldots, a_{n}\right)}, \\
\mu\left(\theta^{\left(a_{1}, a_{2}, \ldots, a_{n}\right)}\right)=\left(\alpha_{1}\left(\theta\left(a_{1}, a_{2}, \ldots a_{n}, u\right), u\right), \ldots, \alpha_{n}\left(\theta\left(a_{1}, a_{2}, \ldots a_{n}, u\right), u\right)\right),
\end{gathered}
$$

where $u$ is an arbitrary element of $A$. Note that the value of each $\alpha_{i}\left(\theta\left(a_{1}, a_{2}, \ldots a_{n}, u\right), u\right)$ does not depend on $u$, since $A$ is right-cancellable. Equality (2.2) implies that

$$
\varepsilon \mu\left(\theta^{\left(a_{1}, a_{2}, \ldots, a_{n}\right)}\right)(u)=\theta^{\left(a_{1}, a_{2}, \ldots, a_{n}\right)}(u) .
$$

Then from Lemma 4.2 we obtain

$$
\varepsilon \mu\left(\theta^{\left(a_{1}, a_{2}, \ldots, a_{n}\right)}\right)=\theta^{\left(a_{1}, a_{2}, \ldots, a_{n}\right)} .
$$

Hence $\left(A, \bar{\theta}\left(A^{n}\right), \varepsilon, \mu\right)$ is an object of $\mathbb{X}_{n}$.

Assume $\varphi: A \rightarrow A^{\prime}$ is a homomorphism of $\mathbb{V}_{n}$-algebras. Consider the mapping $\psi: \bar{\theta}\left(A^{n}\right) \rightarrow \bar{\theta}\left(A^{\prime n}\right)$ sending the bijection $\theta^{\left(a_{1}, a_{2}, \ldots, a_{n}\right)}$ to the one $\theta^{\left(\varphi\left(a_{1}\right), \varphi\left(a_{2}\right), \ldots, \varphi\left(a_{n}\right)\right)}$. Lemma 4.2 implies that it is defined correctly. Moreover, equalities (4.9)-(4.10) and Lemma 4.2 imply that the pair $(\varphi, \psi)$ is a morphism of $\mathbb{X}_{n}$.

Let us now construct the functor $G: \mathbb{X}_{n} \rightarrow R C-\mathbb{V}_{n}$. To this end consider a quadruple (4.13) from $\mathbb{X}_{n}$. Define the following operations on $A$ :

$$
\begin{gathered}
\theta\left(a_{1}, a_{2}, \ldots, a_{n}, b\right)=\varepsilon\left(a_{1}, a_{2}, \ldots, a_{n}\right) b \\
e_{i}=\pi_{i} \mu(e)
\end{gathered}
$$

and

$$
\alpha_{i}(a, b)=\pi_{i} \mu(g)
$$

where $\pi_{i}$ is the canonical projection $A^{n} \rightarrow A$, while $g$ is the unique element of $G$ with $g(b)=a$. Equality (4.14) implies that operations (4.22)-(4.24) give the structure of a $\mathbb{V}_{n}$-algebra on $A$. To show that this algebra is right-cancellable, we observe that

$$
\alpha_{i}\left(\theta\left(a_{1}, a_{2}, \ldots, a_{n}, b\right), \theta\left(a_{1}^{\prime}, a_{2}^{\prime}, \ldots, a_{n}^{\prime}, b\right)=\pi_{i} \mu(g)\right.
$$


where $g$ is the unique element of $G$ with

$$
g\left(\theta\left(a_{1}^{\prime}, a_{2}^{\prime}, \ldots, a_{n}^{\prime}, b\right)=\theta\left(a_{1}, a_{2}, \ldots, a_{n}, b\right) .\right.
$$

Applying (4.22) we obtain

$$
\left(g \varepsilon\left(a_{1}^{\prime}, a_{2}^{\prime}, \ldots, a_{n}^{\prime}\right)\right) b=\varepsilon\left(a_{1}, a_{2}, \ldots, a_{n}\right) b .
$$

Since the action of $G$ is principal, (4.27) implies that

$$
g \varepsilon\left(a_{1}^{\prime}, a_{2}^{\prime}, \ldots, a_{n}^{\prime}\right)=\varepsilon\left(a_{1}, a_{2}, \ldots, a_{n}\right) .
$$

Thus the element $g$ does not depend on $b$, and hence equality (3.1) is satisfied.

If a pair $(\varphi, \psi)$ is a morphism $(A, G, \varepsilon, \mu) \rightarrow\left(A^{\prime}, G^{\prime}, \varepsilon^{\prime}, \mu^{\prime}\right)$, then, as one easily can verify, $\varphi$ is a homomorphism of the corresponding $\mathbb{V}_{n}$-algebras $A \rightarrow A^{\prime}$.

One can verify that $G F=1_{\left(R C-\mathbb{V}_{n}\right)_{*}}$. Moreover, we have a natural isomorphism $\tau: 1_{\mathbb{X}_{n}} \rightarrow F G$. Namely, $\tau_{(A, G, \varepsilon, \mu)}$ is the pair $\left(1_{A}, \kappa\right)$, where $\kappa$ is the homomorphism $G \rightarrow \bar{\theta}\left(A^{n}\right)$ sending $g \in A$ to the mapping $\theta^{\mu(g)}: A \rightarrow A$ (which sends $a \in A$ to $g a$ ).

If $G((A, G, \varepsilon, \mu))$ is a group, then (4.17) obviously is satisfied. The converse statement follows from Lemma 3.4.

\section{Group operations on Right-CANCELlable Protomodular ALGEBRAS}

Let $A$ be a right-cancellable protomodular algebra. As we mentioned above, each $u \in A$ gives the bijection between $A$ and $\bar{\theta}\left(A^{n}\right)$ : it sends an element $a$ of $A$ to the unique $g \in \bar{\theta}\left(A^{n}\right)$ with $g(u)=a$. In fact, this bijection is the composition of the following two bijections

$$
A \stackrel{\gamma_{u}}{\longrightarrow} A^{n} / \sim \longrightarrow \bar{\theta}\left(A^{n}\right)
$$

where $\sim$ is the equivalence relation from Lemma 4.1 , and $\gamma_{u}$ sends an element $a$ of $A$ to the equivalence class of the $n$-tuple

$$
\left(\alpha_{1}(a, u), \alpha_{2}(a, u), \ldots, \alpha_{n}(a, u)\right) .
$$

Transferring the group structure of $\bar{\theta}\left(A^{n}\right)$ via these bijections, we obtain the group structure on $A$. We have

Proposition 5.1. Let $A$ be a right-cancellable protomodular algebra, and let $u$ be its arbitrary element. The binary operation on $A$ defined by:

$$
a \cdot{ }_{u} b=\theta\left(\alpha_{1}(a, u), \alpha_{2}(a, u), \ldots, \alpha_{n}(a, u), b\right) .
$$


is a group operation. Its unit is $u$, while the inverse of an element $a \in A$ under this operation is given by:

$$
a^{-u 1}=\theta\left(\alpha_{1}(u, a), \alpha_{2}(u, a), \ldots, \alpha_{n}(u, a), u\right) .
$$

Proof. Substituting $a_{i}=\alpha_{i}(a, u)$ and $b_{i}=\alpha_{i}(b, u)$ in (4.10), we obtain the following value of $c_{i}$ :

$$
c_{i}=
$$

$\alpha_{i}\left(\theta\left(\alpha_{1}(a, u), \alpha_{2}(a, u), \ldots, \alpha_{n}(a, u), u\right), \theta\left(\alpha_{1}(u, b), \alpha_{2}(u, b), \ldots, \alpha_{n}(u, b), u\right)\right.$, for any $i$. Since $A$ is right-cancellable, we can replace two $u$ 's by $b$ 's in the appropriate positions of the right-hand side of (5.5). Then, from (2.2) we obtain

$$
c_{i}=\alpha_{i}\left(\theta\left(\alpha_{1}(a, u), \alpha_{2}(a, u), \ldots, \alpha_{n}(a, u), b\right), u\right)
$$

Applying the equality

$$
a \cdot{ }_{u} b=\theta_{u}\left(c_{1}, c_{2}, \ldots, c_{n}\right),
$$

we obtain (5.3).

Equality (5.4) can be obtained from (4.9)-(4.10) by similar arguments.

We can give the direct proof of the fact that the binary operation given by (5.3) is indeed a group operation on $A$. To this end let us first give

Lemma 5.2. Let $A$ be a right-cancellable protomodular algebra, and $a, b, c, u$ be its arbitrary elements. For the binary operation $\cdot u$ on $A$ given by (5.3) and any $i(1 \leq i \leq n)$, we have

$$
\alpha_{i}\left(a \cdot{ }_{u} c, b \cdot{ }_{u} c\right)=\alpha_{i}(a, b) .
$$

Proof. We have

$$
\begin{gathered}
\alpha_{i}\left(a \cdot{ }_{u} c, b \cdot{ }_{u} c\right)= \\
\alpha_{i}\left(\theta\left(\alpha_{1}(a, u), \alpha_{2}(a, u), \ldots, \alpha_{n}(a, u), c\right), \theta\left(\alpha_{1}(b, u), \alpha_{2}(b, u), \ldots, \alpha_{n}(b, u), c\right) .\right.
\end{gathered}
$$

Replacing both $c$ 's on the right-hand side of this equality by $u$, and then applying equality $(2.2)$, we obtain $\alpha_{i}(a, b)$.

Now we are ready to give the direct proof of Proposition 5.1.

Proof. Let us introduce the new binary operation on $A$ :

$$
a / u b=\theta\left(\alpha_{1}(a, b), \alpha_{2}(a, b), \ldots, \alpha_{n}(a, b), u\right) .
$$

Applying the fact that $A$ is right-cancellable, identities (2.8) and (2.2), we obtain

$$
\alpha_{i}\left(\left(a /{ }_{u} b\right), u\right)=\alpha_{i}(a, b)
$$


Then

$$
\left(a /{ }_{u} b\right) \cdot{ }_{u} b=\theta\left(\alpha_{1}(a, b), \alpha_{2}(a, b), \ldots, \alpha_{n}(a, b), b\right)=a .
$$

Hence the set $A$ equipped with the binary operations $\cdot_{u}, /{ }_{u}$ and the constant $u$ is a $\mathbb{V}_{1}$-algebra. Moreover, Lemma 5.2 implies that this algebra is right-cancellable. But, as one can easily observe, $a \cdot{ }_{u} u=a$, for any $a \in A$. Therefore, Lemma 3.5 implies that (5.3) is a group operation. Equality (5.4) immediately follows from (5.10).

Example 5.3. For right-cancellable protomodular algebras with $n=1$ (in particular, for left semi-loops) the group operation (5.3) takes the form

$$
a \cdot{ }_{u} b=(a / u) b
$$

while the inverse $a^{-{ }^{1}}$ takes the form $(u / a) u$.

Note that the Mal'cev operation corresponding to the group operation (5.3) is precisely the Mal'cev operation (2.5). Hence we obtain

Corollary 5.4. The Mal'cev term (2.5) is associative on rightcancellable protomodular algebras.

We can summarize the protomodular analogs of the classical group term existence criterion (see Section 1).

Theorem 5.5. Let $\mathbb{V}$ be a variety of universal algebras. The following conditions are equivalent:

(i) the algebraic theory of $\mathbb{V}$ contains a group term;

(ii) the algebraic theory of $\mathbb{V}$ contains protomodular terms with respect to which all $\mathbb{V}$-algebras are right-cancellable;

(iii) the algebraic theory of $\mathbb{V}$ contains protomodular terms $\theta, \alpha_{i}$, and $e_{i}(i=1,2, \ldots, n)$ such that $\theta$ is consociative, and, moreover, $e_{1}=e_{2}=$ $\ldots=e_{n}$;

(iv) the algebraic theory of $\mathbb{V}$ contains protomodular terms $\theta$ and $\alpha_{i}$ $(i=1,2, \ldots, n)$ which satisfy the identity:

$$
\begin{gathered}
\theta\left(\alpha_{1}(a, b), \ldots, \alpha_{n}(a, b), \theta\left(\alpha_{1}(c, d), \ldots, \alpha_{n}(c, d), s\right)\right) \\
=\theta\left(\alpha_{1}\left(\theta\left(\alpha_{1}(a, b), \ldots, \alpha_{n}(a, b), c\right), d\right),\right. \\
\alpha_{2}\left(\theta\left(\alpha_{1}(a, b), \ldots, \alpha_{n}(a, b), c\right), d\right), \ldots, \\
\left.\alpha_{n}\left(\theta\left(\alpha_{1}(a, b), \ldots, \alpha_{n}(a, b), c\right), d\right), s\right) .
\end{gathered}
$$

Proof. The equivalence of (i) and (ii) immediately follows from Proposition 5.1. The equivalence of (i), (iii) and (iv) is given in [16]. 
Remark 5.6. In view of Theorem 5.5 there naturally arises the question whether the notions of a right-cancellable protomodular algebra and of a consociative protomodular algebra are equivalent. The answer is negative. For instance, non-trivial Boolean algebras are consociative with respect to the protomodular terms given in [3] (see Example 3.5 of [16]), but they are not right-cancellable with respect to these terms (see Remark 3.8). Moreover, there are right-cancellable left semi-loops which are not consociative, as follows from Example 3.2.

Finally we will show that right-cancellable $\mathbb{V}_{n}$-algebras can be characterized as groups with simple additional structures. Consider the category $\mathbb{Y}_{n}$ with objects being triples $(A, \sigma, \rho)$, where $A$ is a group, $\sigma$ and $\varrho$ are (not necessarily homomorphisms, but) mappings resp. $A^{n} \rightarrow A$ and $A \rightarrow A^{n}$ such that $\sigma \varrho=1_{A}$.

Proposition 5.7. The category $\left(R C-\mathbb{V}_{n}\right)_{*}$ of right-cancellable $\mathbb{V}_{n}$ algebras with fixed elements is isomorphic to the category $\mathbb{Y}_{n}$. Under this isomorphism, the strict right-cancellable $\mathbb{V}_{n}$-algebras (with fixed elements) correspond precisely to the triples $(A, \sigma, \rho)$ where $\sigma$ and $\varrho$ are bijections. If $n=1$, then the groups (with fixed elements) correspond precisely to the triples $(A, \sigma, \rho)$ with $\sigma=\varrho=1_{A}$.

Proof. Let us construct the functor $P:\left(R C-\mathbb{V}_{n}\right)_{*} \rightarrow \mathbb{Y}_{n}$. If $A$ is a right-cancellable $\mathbb{V}_{n}$-algebra and $u \in A$, then define $P(A)$ as the triple $\left(A, \theta_{u},\left(\alpha_{1}(-, u), \alpha_{2}(-, u), \ldots, \alpha_{n}(-, u)\right)\right)$, where $A$ is equipped with the group operation $\cdot{ }_{u}$.

To construct the functor $Q: \mathbb{Y}_{n} \rightarrow\left(R C-\mathbb{V}_{n}\right)_{*}$, consider a triple $(A, \sigma, \varrho)$ from $\mathbb{Y}_{n}$. Define the following operations on $A$ :

$$
\begin{gathered}
\theta\left(a_{1}, a_{2}, \ldots, a_{n}, b\right)=\sigma\left(a_{1}, a_{2}, \ldots, a_{n}\right) b, \\
\alpha_{i}(a, b)=\pi_{i} \varrho\left(a b^{-1}\right), \\
e_{i}=\pi_{i} \varrho(e) .
\end{gathered}
$$

One can easily show that the set $A$ equipped with these operations and with the fixed element $e$ is an object of the category $\left(R C-\mathbb{V}_{n}\right)_{*}$.

To verify that the functors $P$ and $Q$ are mutually inverse, let us observe that for an algebra $A$ from $R C-\mathbb{V}_{n}$, elements $a, b$ and $u$ from $A$ and the operation $\alpha_{i}^{\prime}$ of $Q P(A)$, we have

$$
\alpha_{i}^{\prime}(a, b)=\rho_{i}\left(a \cdot{ }_{u} b^{-1}\right)=\alpha_{i}\left(\left(a \cdot{ }_{u} b^{-1}\right), u\right) .
$$

Proposition 5.1 and Lemma 5.2 imply that the right-hand part of (5.17) is precisely $\alpha_{i}(a, b)$.

If the algebra corresponding to an object $(A, \sigma, \rho)$ from $\mathbb{Y}_{n}$ is a group, then, for any $a \in A$, we have $a=\alpha(a, e)=\rho(a)$. Hence $\rho=\varepsilon=1_{A}$. 
Proposition 5.7 in particular implies Proposition 3.7. In fact, we can make the latter statement stronger.

Proposition 5.8. Let $A$ be a finite algebra from a variety $\mathbb{V}$ that has protomodular terms with $n=1$. If $A$ is not a left semi-loop with respect to these protomodular terms, then the algebraic theory of $\mathbb{V}$ does not contain a term whose corresponding operation on $A$ is a group operation.

Proof. For convenience, we use the abbreviation $a b$ for $\theta(a, b)$, and $a / b$ for $\alpha(a, b)$. There are elements $a, b \in A$ with

$$
(a b) / b \neq a \text {. }
$$

Let $I$ be the ideal generated by $a$ and $(a b) / b$. Consider the mapping $\theta_{b}: A \rightarrow A$ sending $a$ to $a b$. The restriction of $\theta_{b}$ on $I$ is not injective, as follows from (5.18) and the equality

$$
((a b) / b) b=a b .
$$

Hence, since $I$ is finite, the cardinality of $\theta_{b}(I)$ is less than the cardinality of $I$. But $\theta_{b}(I)=I b$ is an equivalence class of the congruence induced by $I$ (see Section 2). This completes the proof.

\section{ACKNOWLEDGMent}

Financial support from Shota Rustaveli National Science Foundation (Ref.: FR-18-10849) is gratefully acknowledged.

\section{REFERENCES}

[1] R. Bëlohlavek, I. Chajda, Congruences and ideals in semiloops, Acta Sci. Math. (Szeged), 59(1994), 43-47.

[2] E. Beutler, An idealtheoretic characterization of varieties of abelian $\Omega$-groups, Algebra Universalis 8(1978), 91-100.

[3] F. Borceux, M. M. Clementino, Topological semi-abelian algebras, Adv. Math, 190(2005), 425-453.

[4] F. Borceux, M. M. Clementino, Topological protomodular algebras,Topology and Its Appl., 153(2006), 3085-3100.

[5] D. Bourn, Normalization equivalence, kernel equivalence and affine categories, Lecture notes in Math, 1448(1991), 43-62.

[6] D. Bourn, G. Janelidze, Characterization of protomodular varieties of universal algebra, Theory Appl. Categ., 11(2002), 143-147.

[7] J. Certaine, The ternary operation $(a b c)=a b^{-1} c$ of a group, Bull. Amer. Math. Soc. 49(1943), 869-877.

[8] O. Chein, H. O. Pflugfelder, J. D. H. Smith, eds. Quasigroups and loops: theory and applications. Berlin: Heldermann, 1990.

[9] J.B. Fraleigh, A first course in abstract algebra, 7th edn. Pearson, University of Rhode Island, 2003. 
[10] P. Gumm, A. Ursini, Ideals in universal algebras, Algebra Universalis 19(1984), 45-54.

[11] G. Janelidze, L. Márki, W. Tholen, Semi-abelian categories, J. Pure Appl. Algebra, 168(2002), 367-386.

[12] P. T. Johnstone, A note on the semiabelian variety of Heyting semilattice. In: Galois theory, Hopf algebras, and Semiabelian categories, Fields Inst. Commun. 43(2004), 317-318.

[13] P. T. Johnstone, M. C. Pedicchio, Remarks on continuous Mal'cev algebras, Rend. Instit. Mat. Univ. Trieste. 25 (1995), 277-287.

[14] A. Ursini, Osservazioni sulla varieta BIT, Boll. U. M. I. 8(1973), 205-211.

[15] A. Ursini, On subtractive varieties, I, Algebra Universalis 31(1994), 204-222.

[16] D. Zagurashvili, Associativity-like conditions on protomodular algebras, Algebra Universalis 81:1(2020).

Authors address: Andrea Razmadze Mathematical Institute of Tbilisi State University, 6 Tamarashvili Str., Tbilisi 0177, Georgia, e-mail: dali.zangurashvili@tsu.ge 